\title{
DESEMPENHO DE DOZE ESPÉCIES ARBÓREAS NATIVAS E INTRODUZIDAS COM POTENCIAL DE USO MÚLTIPLO NO ESTADO DO ACRE, BRASIL ${ }^{1}$
}

\author{
Elias Melo de MIRANDA², Judson Ferreira VALENTIM ${ }^{3}$
}

\begin{abstract}
RESUMO - O objetivo deste estudo foi avaliar o desempenho de espécies arbóreas com potencial de uso múltiplo, nas condições ambientais da Amazônia ocidental brasileira, visando recomendálas como componentes de sistemas agroflorestais e para recuperação de áreas degradadas. O experimento foi estabelecido na Estação Experimental da Embrapa Acre, com 12 espécies nativas e introduzidas, no espaçamento de $1,50 \mathrm{~m}$ entre linhas, por $1,50 \mathrm{~m}$ entre plantas. $\mathrm{O}$ delineamento experimental foi de blocos casualizados, com quatro repetições. Cada espécie foi representada por cinco plantas em cada repetição. As avaliações, realizadas até quatro anos e meio após o plantio mostraram que: a) todas as espécies apresentaram indices de sobrevivência superiores a $80 \%$, com exceção de Cedrela odorata (75\%), Aspidosperma vargasii (65\%) e Spondias lutea (65\%); b) o incremento médio anual em altura total foi maior em Acacia mangium (2,91 m) e Acacia pollyphylla (2,66 m), seguida por Leucaena leucocephala, Calycophyllum spruceanum e Gliricidia sepium com médias de 2,38, 1,56 e 1,40 m, respectivamente; c) o incremento médio anual em diâmetro basal também foi maior em $A$. mangium $(4,98 \mathrm{~cm})$ e A. pollyphylla $(3,92 \mathrm{~cm})$, seguidas de Erythrina fusca $(3,06 \mathrm{~cm})$, Spondias lutea $(2,37 \mathrm{~cm})$, Erythrina poeppigiana $(2,17 \mathrm{~cm})$ e $E$. berteroana $(2,12 \mathrm{~cm})$; d) o incremento anual em diâmetro da copa foi maior em $A$. pollyphylla $(2,50 \mathrm{~m})$, G. sepium $(1,03$ $\mathrm{m})$, Torresea acreana $(0,98 \mathrm{~m})$ e Erythrina fusca $(0,87 \mathrm{~m})$. Estes resultados sugerem a boa adaptação das espécies introduzidas que apresentaram desenvolvimento superior à maioria das nativas, principalmente em termos de capacidade de estabelecimento e crescimento inicial.
\end{abstract}

Palavras-chave: Amazônia, árvores de uso múltiplo, Brasil, sistemas agroflorestais, áreas degradadas.

Performance of Twelve Native and Introduced Tree Species with Multiple Use Potential in the State of Acre, Brazil

ABSTRACT - The objective of this study was to evaluate the performance of tree species with multiple use potential, in the local environmental conditions of the western Brazilian Amazon, in order to recommend these species as components of agroforestry systems and for reclamation of degraded areas. The experiment was established at the Experimental Station of Embrapa Acre, with 12 native and introduced species, using 1.5 by $1.5 \mathrm{~m}$ spacing between rows and plants. The experimental design was a randomized complete block, with four replications and five plants in each replication. The evaluations at four and a half years after planting showed that: a) all species presented survival indexes above $80 \%$, with exception of Cedrela odorata $(75 \%)$, Aspidosperma varyasii $(65 \%)$ and Spondias lutea $(65 \%)$; b) the mean annual increment in total height was greater in Acacia mangium $(2.91 \mathrm{~m})$ and Acacia pollyphylla $(2.66 \mathrm{~m})$, followed by Leucaena leucocephala, Calycophyllum spruceanum and Gliricidia sepium, with means of $2.38 \mathrm{~m}, 1.56 \mathrm{~m}$ and $1.40 \mathrm{~m}$, respectively; c) the mean annual increment in basal diameter was greater in Acacia mangium (4.98 $\mathrm{cm})$ and Acacia pollyphylla $(3.92 \mathrm{~cm})$, followed by E. fusca $(3.06 \mathrm{~cm})$, Spondias lutea $(2.37 \mathrm{~cm})$, Erythrina poeppigiana $(2.17 \mathrm{~cm})$ and E. berteroana $(2.12 \mathrm{~cm})$; d) the annual increment in tree crown diameter was greater in A. pollyphylla $(2.50 \mathrm{~m})$, G. sepium $(1.03 \mathrm{~m})$, Torresea acreana $(0.98$ $\mathrm{m})$ and $E$. fusca $(0.87 \mathrm{~m})$. These results suggest good adaptation of the introduced species, which presented development superior to most of the native species, especially in terms of capacity for establishment and initial growth.

Key-words: agroforestry systems, amazon, Brazil, degraded areas, multipurpose trees.

\footnotetext{
${ }^{1}$ Trabalho realizado com apoio financeiro do GEF, por meio do projeto ASB/IGRAF.

${ }^{2}$ Eng.-Agr., M.Sc., Embrapa Acre, Caixa Postal 392, CEP 69908-970, Rio Branco, AC. e-mail: elias@cpafac.embrapa.br

${ }^{3}$ Eng.-Agr., Ph.D., Embrapa Acre.
} 


\section{INTRODUÇÃO}

A rapidez do processo de desmatamento na América tropical é motivo de grande preocupação. A diminuição da cobertura de ecossistemas florestais tropicais e de bacias hidrográficas, somada a uma diminuição da importância das árvores nos sistemas agrícolas, gera, de forma crescente, sistemas de produção e desenvolvimento não sustentáveis. Mais preocupante ainda é a falta de manejo, de forma sustentada, das florestas primárias e secundarias existentes e a reposição das mesmas (Flores Rodas, 1989).

Os sistemas agroflorestais podem contribuir para solucionar estes problemas, devido as funções biológicas e sócio-econômicas que podem cumprir. A presença de árvores favorece os sistemas de produção, em aspectos tais como a manutenção da ciclagem de nutrientes e o aumento na diversidade de espécies (Organizacion de Estudios Tropicales e Centro Agronómico Tropical de Investigación y Enseñanza, 1986).

As necessidades e justificativas para o reflorestamento, especialmente com árvores de uso múltiplo em monocultivo ou em sistemas agroflorestais, são muitas e variam de acordo com as características ambientais e sócio-econômicas de cada região. No Acre, a cobertura vegetal predominante é a floresta tropical úmida, com elevada diversidade de espécies por hectare. Nestas áreas, a principal atividade econômica, no último século, tem sido o extrativismo do látex da seringueira (Hevea sp) e a coleta da castanha-do-brasil (Bertholetia excelsa H.B.K). Entretanto, nos últimos 30 anos, a desestruturação do sistema extrativo da borracha dos seringais nativos, a criação de projetos de colonização e a implantação e expansão da atividade pecuária transformaram a economia do setor primário do Acre (Valentim, 1989; Valentim \& Moreira, 1994).

Segundo dados da Fundação de Tecnologia do Estado do Acre (1989), o Estado apresentava, àquela época, $4,89 \%$ da sua área total desmatada. Dados mais recentes, apontam uma área desmatada de cerca de $9 \%$, o que corresponde aproximadamente a 1,37 milhões de hectares (INPE, 1998). Os sistemas de produção desenvolvidos nestas áreas apresentam problemas graves, relacionados a sustentabilidade econômica, social e ambiental (Homma, 1993; Kitamura, 1994).

Os sistemas agroflorestais são alternativas de uso da terra, que podem ser ecológica e economicamente viáveis na Amazônia. Para isso, devem ser bem planejados, sendo um dos aspectos importantes a identificação de espécies lenhosas, adequadas para serem usadas como componentes desses sistemas.

Este trabalho teve como objetivo avaliar o desempenho de espécies arbóreas com potencial de uso múltiplo, nas condições ambientais locais, visando recomendar, a curto prazo, espécies arbóreas de uso múltiplo, adaptadas às condições edafoclimáticas locais, para serem utilizadas na 
recuperação de áreas degradadas e como componentes de vários tipos de sistemas, produzindo bens e serviços, tais como: cercas vivas, sombreamento de culturas perenes e de animais, produção de forragem, adubo verde, madeira e frutos.

\section{MATERIAL E MÉTODOS}

Este estudo foi desenvolvido na Estação Experimental da Embrapa Acre, localizada no $\mathrm{km} 14$ da rodovia BR 364, em Rio Branco, AC. O ecossistema da região é de floresta tropical úmida e a altitude é de $160 \mathrm{~m}$. A área foi desmatada no início da década de 70 , sendo estabelecida pastagem de colonião (Panicum maximum). Durante a década de 80 , a área foi utilizada para experimentos diversos de hortaliças e forrageiras e posteriormente mantida em pousio por cerca de três anos.

O solo da área é do tipo Podzólico Vermelho Amarelo, com as seguintes características físicoquímicas: areia grossa $147 \mathrm{~g} \mathrm{~kg}^{-1}$; areia fina $474 \mathrm{~g} \mathrm{~kg}^{-1}$; silte $267 \mathrm{~g} \mathrm{~kg}^{-1}$; argila $112 \mathrm{~g} \mathrm{~kg}^{-1} ; \mathrm{pH}=5,6 ; \mathrm{P}=3 \mathrm{mg} \mathrm{kg}^{-1}$; $\mathrm{K}^{+}=0,93 \mathrm{mmol}_{\mathrm{c}} \mathrm{dm}^{-3} ; \mathrm{Ca}^{++}=20$ $\mathrm{mmol}_{\mathrm{c}} \mathrm{dm}^{-3} ; \mathrm{Mg}^{++}=6 \mathrm{mmol}_{\mathrm{c}} \mathrm{dm}^{-3}$; $\mathrm{Na}^{+}=0,4 \mathrm{mmol}_{\mathrm{c}} \mathrm{dm}^{-3} ; \mathrm{Al}^{+++}=2,8$ $\mathrm{mmol}_{\mathrm{c}} \mathrm{dm}^{-3} ; \mathrm{H}^{+}+\mathrm{Al}^{+++}=21,8 \mathrm{mmol}_{\mathrm{c}}$ $\mathrm{dm}^{-3}$; e M. O. $=14,6 \mathrm{~g} \mathrm{~kg}^{-1} \cdot \mathrm{A}$ precipitação média anual é de 1865 mm e a temperatura média anual é de $24,3^{\circ} \mathrm{C}$.

O delineamento experimental foi de blocos casualizados, com quatro repetições. Foram avaliadas 12 espécies, sendo seis nativas: Amarelão
(Aspidosperma vargasii A.DC.), Cedro (Cedrela odorata L.), Cerejeira (Torresea acreana Duck.), Mulateiro (Calycophyllum spruceanum Bent.), Taperebá (Spondias lutea L.) e Espinheiro Preto (Acacia pollyphylla A.DC.); e seis introduzidas: Gliricidia sepium (Jacquin) Kunth ex Walpers, Erythrina fusca Lour., Erythrina poeppigiana (Walp.) O.F. Cook, Erythrina berteroana Urban, Leucaena leucocephala (Lam. De Wit.) e Acacia mangium Willd. As parcelas foram constituídas por linhas de cinco plantas de cada espécie, plantadas no espaçamento de $1,50 \mathrm{x}$ $1,50 \mathrm{~m}$. Foram utilizadas como bordadura, duas fileiras de $L$. leucocephala ao redor de cada bloco. As mudas foram preparadas em sementeira, sendo posteriormente repicadas para sacos plásticos de $15 \mathrm{x}$ $30 \mathrm{~cm}$ e plantadas no local definitivo em janeiro de 1993.

As avaliações foram realizadas com base na determinação da sobrevivência e de observações anuais do crescimento, através da medição da altura total, diâmetro basal, diâmetro da copa e diâmetro a altura do peito (DAP) até quatro anos e meio de idade das plantas. Também foram realizadas observações sobre $\mathrm{o}$ ataque de pragas $\mathrm{e}$ doenças, bem como do início da fase reprodutiva das espécies. Para a análise estatística adotou-se o procedimento GLM (General Linear Models Procedure) do programa estatístico SAS Software, Release 6.12 (SAS Institute Inc., Cary, NC, USA, 1989-1996).

RESULTADOS E DISCUSSÃO 
A sobrevivência da maioria das espécies foi elevada, observando-se que apenas as espécies Spondias lutea e Aspidosperma vargasii apresentaram taxas de mortalidade superiores a $30 \%$. As espécies mostraram os seguintes percentuais de sobrevivência aos 12 meses de idade: Leucaena leucocephala, 100\%; Erythrina fusca, $100 \%$; Calycophyllum spruceanum, 100\%; Gliricidia sepium, 95\%; Torresea acreana, 95\%; Erythrina berteroana, 90\%; Acacia pollyphylla, 90\%; Erythrina poeppigiana, 90\%; Acacia mangium, $80 \%$; Cedrela odorata, $75 \%$; A. vargasii, $65 \%$; e S. lutea, $65 \%$.

$\mathrm{O}$ crescimento das espécies foi discutido com base nos resultados da última avaliação, realizada em 1997 , quando as plantas estavam com quatro anos e meio de idade. Foram utilizados, também, dados de incremento médio anual das variáveis avaliadas no periodo (1994 a 1997).

As espécies que apresentaram maior incremento médio em altura total, no período avaliado, foram $A$. mangium $(2,91 \mathrm{~m})$, A. pollyphylla $(2,66 \mathrm{~m})$ e L. leucocephala $(2,38 \mathrm{~m})$ (Tab. 1). Quanto ao incremento em diâmetro basal, também destacaram-se as espécies Acacia mangium, com um notável incremento médio anual de $4,98 \mathrm{~cm}$ e $A$. pollyphylla (Espinheiro Preto) com $3,92 \mathrm{~cm}$, seguidas por $E$. fusca com 3,06 cm e Spondias lutea com $2,37 \mathrm{~cm}$. E. poeppigiana e $E$. berteroana apresentaram incremento superior a 2,00 cm (Tab. 2).

A variável diâmetro de copa só foi avaliada até o ano de 1996, pois após esta data, as copas de algumas espécies já encontravam-se bastante fechadas, dificultando as medições. As espécies que apresentaram maiores incrementos foram A. pollyphylla com $2,50 \mathrm{~m}$, Gliricidia sepium com 1,03 m e Torresea acreana com $\quad 0,98 \mathrm{~m}$ (Tab. 3).

A espécie que apresentou a maior velocidade de crescimento foi a A. mangium, considerando-se as variáveis altura total, diâmetro basal e diâmetro à altura do peito (DAP). As médias obtidas para estas variáveis, foram significativamente superiores às médias das demais espécies (Tabs. 1, 2 e 3).

Em altura total, as espécies que apresentaram maior crescimento médio foram $A$. mangium com 11,72 m, A. pollyphylla com $9,29 \mathrm{~m}$ e $L$. leucocephala com 7,23 m. As médias obtidas diferiram significativamente entre si e foram superiores às obtidas para as demais espécies (Tab. 1). Analisando-se o crescimento em diâmetro basal (Tab. 2), destacaram-se A. mangium $(19,75 \mathrm{~cm})$, E. fusca $(16,31 \mathrm{~cm})$ e A. pollyphylla $(13,95 \mathrm{~cm})$. Quanto ao DAP, medido a partir de 1997, estas espécies apresentaram o seguinte desempenho: $14,98 \mathrm{~cm}, 9,04$ $\mathrm{cm}$ e $9,65 \mathrm{~cm}$, respectivamente (Tab. 3).

$O$ crescimento em altura de $L$. leucocephala e G. sepium obtido neste experimento está de acordo com os valores encontrados por Hughell (1990), ao desenvolver modelos de predição de crescimento e rendimento para estas duas espécies em solos de regular qualidade na América Central (Centro Agronómico Tropical de Investigación y Enseñanza, 1991a, 
Tabela 1. Altura total $(\mathrm{m})$ e os respectivos incrementos médios verificados no crescimento de espécies multi-uso, nativas e introduzidas, no período de 1994 a 1997, em Rio Branco-AC.

\begin{tabular}{lccccc}
\hline Espécie & 1994 & 1995 & 1996 & $1997^{1}$ & Incr. Médio \\
\hline Acacia mangium & 2,87 & 5,64 & 9,78 & $11,72 \mathrm{a}$ & 2,91 \\
Acacia pollyphylla & 1,32 & 2,55 & 7,12 & $9,29 \mathrm{~b}$ & 2,66 \\
Aspidosperma vargasii & 0,25 & 0,48 & 1,06 & $1,67 \mathrm{~g}$ & 0,47 \\
Calycophyllum spruceanum & 1,27 & 2,46 & 4,97 & $5,94 \mathrm{~d}$ & 1,56 \\
Cedrela odorata & 0,43 & 1,19 & 2,40 & $2,66 \mathrm{f}$ & 0,74 \\
Erythrina berteroana & 1,48 & 2,42 & 3,21 & $3,84 \mathrm{e}$ & 0,78 \\
Erythrina fusca & 2,09 & 3,17 & 4,39 & $5,53 \mathrm{~d}$ & 1,35 \\
Erythrina poeppigiana & 1,48 & 2,42 & 3,78 & $4,63 \mathrm{e}$ & 0,85 \\
Gliricidia sepium & 1,66 & 3,15 & 4,95 & $5,87 \mathrm{~d}$ & 1,40 \\
Leucaena leucocephala & 3,07 & 4,20 & 6,04 & $7,23 \mathrm{C}$ & 2,38 \\
Spondias lutea & 0,95 & 1,94 & 3,73 & $4,43 \mathrm{e}$ & 1,16 \\
Torresea acreana & 1,36 & 2,02 & 2,53 & $3,77 \mathrm{e}$ & 0,80 \\
\hline
\end{tabular}

'Médias seguidas da mesma letra, na coluna indicada, nấo diferem estatisticamente entre si, pelo teste de Duncan, ao nível de $5 \%$ de probabilidade.

1991b).

Acacia mangium foi considerada, isoladamente, a espécie mais promissora, em termos de velocidade de crescimento. Esta espécie mostrou excelente desempenho no campo, observando-se plantas vigorosas e de crescimento uniforme. Os resultados obtidos para A. mangium, levando-se em conta o tipo de solo onde o experimento foi estabelecido, são coerentes com os relatados por Centro Agronómico Tropical de Investigacion y Enseñanza (1992), que afirma que "em solos de boa qualidade o crescimento desta espécie é surpreendente: mais de quatro metros de altura e acima de cinco centímetros 
Tabela 2. Diâmetro basal $(\mathrm{cm})$ e os respectivos incrementos médios verificados no crescimento de espécies multi-uso, nativas e introduzidas, no período de 1994 a 1997, em Rio Branco-AC.

\begin{tabular}{lccccc}
\hline Espécie & 1994 & 1995 & 1996 & $1997^{1}$ & Incr. Médio \\
\hline Acacia mangium & 4,82 & 8,03 & 16,60 & $19,75 \mathrm{a}$ & 4,98 \\
Acacia pollyphylla & 2,20 & 3,86 & 10,16 & $13,95 \mathrm{c}$ & 3,92 \\
Aspidosperma vargasii & 0,79 & 1,05 & 2,41 & $3,42 \mathrm{f}$ & 0,88 \\
Calycophyllum spruceanum & 2,36 & 3,39 & 5,91 & $6,63 \mathrm{e}$ & 1,77 \\
Cedrela odorata & 1,45 & 2,43 & 5,20 & $6,42 \mathrm{e}$ & 1,65 \\
Erythrina berteroana & 4,97 & 7,67 & 9,40 & $11,33 \mathrm{~d}$ & 2,12 \\
Erythrina fusca & 7,12 & 9,37 & 14,05 & $16,31 \mathrm{~b}$ & 3,06 \\
Erythrina poeppigiana & 4,37 & 6,01 & 8,98 & $10,90 \mathrm{~d}$ & 2,17 \\
Gliricidia sepium & 3,47 & 5,58 & 7,91 & $9,39 \mathrm{~d}$ & 1,97 \\
Leucaena leucocephala & 2,78 & 4,35 & 6,15 & $6,85 \mathrm{e}$ & 1,35 \\
$\begin{array}{l}\text { Sorresea acreana } \\
\text { Spondias lutea }\end{array}$ & 2,32 & 3,57 & 6,92 & $9,43 \mathrm{~d}$ & 2,37 \\
\hline
\end{tabular}

'Médias seguidas da mesma, letra na coluna indicada, năo diferem estatisticamente entre si, pelo teste de Duncan, ao nível de $5 \%$ de probabilidade.

de diâmetro por ano".

De todas as espécies avaliadas, somente $L$. leucocephala apresentou floração e frutificação aos 12 meses de idade, estando de acordo com o reportado por Centro Agronómico Tropical de Investigacion y Enseñanza (1991b), em plantios sob condições apropriadas de clima e solos. $G$. sepium e E. berteroana atingiram a fase reprodutiva após completarem dois anos de idade e $C$. spruceanum aos três anos. As demais espécies não chegaram à maturação sexual durante 
Tabela 3. Diâmetro de copa $(\mathrm{m})$, incrementos médios verificados no crescimento de espécies multi-uso, nativas e introduzidas, no periodo de 1994 a 1996, e o DAP $(\mathrm{cm})$ medido a partir de 1997, em Rio Branco-AC.

\begin{tabular}{|c|c|c|c|c|c|}
\hline Espécie & 1994 & 1995 & $1996^{1}$ & Incr. Médio & DAP1 (1997) \\
\hline Acacia mangium & 1,74 & 2,69 & $3,11 \mathrm{~b}$ & 0,68 & $14,98 \mathrm{a}$ \\
\hline Acacia pollyphylla & 1,07 & 2,25 & $6,07 a$ & 2,50 & $9,65 a$ \\
\hline Aspidospema vargasii & $\mathrm{nm}$ & 0,37 & $1,21 d$ & 0,60 & $1,79 \mathrm{~g}$ \\
\hline Calycophyllum spruceanum & 0,61 & 0,93 & $1,82 \mathrm{~cd}$ & 0,60 & $4,50 \mathrm{def}$ \\
\hline Cedrela odorata & 0,42 & 0,82 & $1,20 d$ & 0,39 & 3,81 ef \\
\hline Erythrina berteroana & 1,07 & 2,44 & $2,56 b c$ & 0,74 & $4,86 \mathrm{def}$ \\
\hline Erythrina fusca & 1,46 & 2,23 & $3,20 \mathrm{~b}$ & 0,87 & $9,04 b$ \\
\hline Erythrina poeppigiana & 0,73 & 1,37 & $1,66 d$ & 0,46 & $6,75 \mathrm{c}$ \\
\hline Glincidia sepium & 1,14 & 2,28 & $3,21 b$ & 1,03 & 5,18 de \\
\hline Leucaena leucocephala & 1,61 & 2,37 & $2,82 b$ & 0,60 & 4,96def \\
\hline Spondias lutea & 0,44 & 1,16 & $1,74 \mathrm{~cd}$ & 0,65 & $5,51 c d$ \\
\hline Torresea acreana & $\mathrm{nm}$ & 1,15 & $1,97 \propto d$ & 0,98 & $3,66 f$ \\
\hline
\end{tabular}

'Médias seguidas da mesma letra, nas colunas indicadas, não diferem estatisticamente entre si, pelo teste de Duncan, ao nivel de $5 \%$ de probabilidade. nm=não medido (sem copa).

o período de avaliações.

Nas observações realizadas sobre a ocorrência de pragas e doenças, verificou-se a ocorrência de um lepidóptero de hábito noturno, causando broca nos ramos das três espécies do gênero Erythrina, ocasionando a morte dos ponteiros. $\mathrm{O}$ referido inseto foi identificado como Terastia meticulosellus (Lep.: Pyralidae), que todavia parece pouco afetar o crescimento vegetativo das plantas de Erythrina. Na Costa Rica, Hilje et al. (1993) relatam a ocorrência 
desta espécie, causando os mesmos danos aos plantios locais. Afirmam, entretanto, que ainda não alcançou importância econômica naquele país, mas que poderiam alcançar, se as espécies de Erythrina forem amplamente plantadas como um componente em novos sistemas agroflorestais.

Entre as espécies nativas, os melhores resultados foram obtidos com o Espinheiro Preto ( $A$. pollyphylla) e Mulateiro ( $C$. spruceanum). No caso do Mulateiro, obteve-se um crescimento em altura comparável ao relatado por Oliveira et al. (1992), que registraram uma altura média de $0,88 \mathrm{~m}$, aos 11 meses, em plantios homogêneos em Rio Branco, AC. Estes autores observaram uma alta variabilidade no crescimento desta espécie, também verificada com as espécies nativas incluidas no presente estudo, por se tratarem de materiais desconhecidos e geneticamente variados.

Durante a condução do experimento, ocorreu problema de excesso de competição entre algumas espécies, já que no momento da aleatorização das parcelas, preferiu-se não levar em conta as diferenças de hábito de crescimento entre as espécies avaliadas. Desta forma, ocorreram alguns casos de parcelas de espécies de rápido crescimento e de maior porte ficarem ao lado de parcelas com espécies de crescimento inicial lento, o que deve ter influenciado no desenvolvimento de algumas dessas espécies, não permitindo a manifestação de todo o seu potencial. Todavia, os princípios da aleatorização e repetição adotados contribuiram para diluir estes efeitos no experimento.

As espécies introduzidas avaliadas neste trabalho já são amplamente utilizadas em grande parte das regiões tropicais do mundo, na recuperação de áreas degradadas, em plantações isoladas, ou como componentes de sistemas agroflorestais. A maioria está adaptada a amplas condições de solo e clima, apresentando rendimentos elevados. Uma exceção deve ser feita para $L$. leucocephala, que em solos excessivamente ácidos tem seu desenvolvimento limitado.

A maioria das espécies nativas apresentaram indivíduos vigorosos e de bom crescimento, podendo através da propagação vegetativa e de melhoramento genético reduzir a desuniformidade observada no crescimento. Inicialmente, pode-se obter um aumento no rendimento destas espécies com a simples aplicação de técnicas de coleta de sementes visando aumentar a qualidade do material coletado.

\section{CONCLUSÕES}

As espécies introduzidas apresentaram, no geral, melhor desempenho em relação ao estabelecimento, crescimento e uniformidade, quando comparado com as espécies nativas.

As espécies introduzidas foram promissoras para usos diversos, nas condições ambientais locais, 
mostrando boa adaptação, com crescimento satisfatório e sem problemas fitossanitários que venham a limitar seu desenvolvimento.

Com base no desempenho apresentado e no uso potencial, as espécies Acacia mangium, Leucaena leucocephala, Gliricidia sepium, Erythrina fusca, E. poeppigiana, $E$. berteroana (introduzidas) e Acacia pollyphylla, Calycophyllum spruceanum e Spondias lutea (nativas) são recomendadas para o plantio na região.

\section{Bibliografia citada}

Centro Agronómico Tropical de Investigacion y Enseñanza. 1991a. Madreado (Gliricidia sepium): especie de árbol de uso múltiple en América Central. CATIE, Turrialba, C.R. 72p. (CATIE. Série Técnica. Informe técnico, 180).

Centro Agronómico Tropical de Investigacion y Enseñanza. 1991 b. Leucaena (Leucaena leucocephala): especie de árbol de uso múltiple en América Central. CATIE, Turrialba, C.R. 60p. (CATIE. Série Técnica. Informe técnico, 166).

Centro Agronómico Tropical de Investigacion y Enseñanza. 1992. Mangium (Acacia mangium): especie de árbol de uso múltiple en América Central. CATIE, Turrialba, C.R. 58p. (CATIE, Série Técnica. Informe técnico, 196).

Flores Rodas, J.G. 1989. Problemática de la reforestación en América latina. In: Centro Agronómico Tropical de Investigacion y Enseñanza. Curso Centroamericano de Silvicultura de Plantaciones de Espécies de Arboles de Uso Multiple. CATIE, Turrialba, C.R. np. (Informe interno).

Fundação de Tecnologia do Estado do Acre. 1989. Monitoramento da cobertura florestal do Estado do Acre: desmatamento e uso atual da terra.
FUNTAC, Rio Branco. 21p.

Homma, A.K.O. 1993. Extrativismo vegetal na Amazônia: limites e oportunidades. EMBRAPA-SPI. Brasilia. 202 p.

Hughell, D.A. 1990. Modelos para la predicción del crecimiento y rendimiento de cuatro especies de árboles de uso múltiple en América Central. CATIE, Turrialba, C.R. 70p. (CATIE. Série Técnica. Boletin Técnico, 22).

Hilje, L.; Shannon, P.J.; Coto, D. 1993. Insects associated with Erythrina species in Costa Rica. In: Westley, S.B.; Powell, M.H. (Eds.). Erythrina in the new and old worlds. Nitrogen fixing tree association, Hawaii, USA. p.330-339.

INPE. Divulgação das estimativas oficiais do desflorestamento bruto na Amazônia Brasileira 1995, 1996 e 1997. Disponivel: site inpe (23 de junho de 1998). http/l www.inpe.br/amz-04.htm. Consultado em 22 set. 1998.

Kitamura, P.C. 1994. A Amazônia e o desenvolvimento sustentável. EMBRAPASPI. Brasilia. $182 \mathrm{p}$.

Oliveira, M.V.N.d'; Mendes, I.M. da S.; Silveira, G. da S. 1992. Estudo do Mulateiro, Calycophyllum spruceanum Benth, em condições de ocorrência natural e em plantios homogèneos. EmbrapaCPAF/AC, Rio Branco. 17p. (EmbrapaCPAF/AC. Boletim de Pesquisa, 8).

Organizacion de Estudios Tropicales; Centro Agronómico Tropical de Investigacion y Ensenanza. 1986. Sistemas agroforestales: principios y aplicaciones en los tropicos. OTS/ CATIE, San José, Costa Rica, p.99-128.

Valentim, J.F. 1989. Impacto ambiental da pecuária no Acre. In: EMBRAPA. Centro de Pesquisa Agroflorestal do Acre (Rio Branco,AC); Instituto do Meio Ambiente de Acre. Documento base do Curso de Avaliação do Impacto ambiental da Pecuária no Acre. Embrapa-CPAF/AC, Rio Branco, Acre. 33p.

Valentim, J.F.; Moreira, P. 1994. Adaptação, produtividade, composição morfológica e distribuição estacional da produção de 
forragem de ecotipos de Panicum maximum no Acre. Embrapa-CPAF/AC, Rio Branco, Acre. 17p. (Embrapa CPAF/AC.

Boletim de Pesquisa, 11). 